\title{
Corona im Pflegeheim. Ein Erfahrungsbericht aus der Praxis
}

Barbara Derler und Brigitte Pichler - befragt von Johann Platzer

Im Rahmen der aktuellen „Corona-Krise“ ist vor allem den Pflegewohnhäusern eine hohe Aufmerksamkeit zuteilgeworden. Wie in vielen anderen Ländern kam es auch in Österreich in Alten- und Pflegeheimen zu ClusterHäufungen von Covid-19-Erkrankungen. Davon waren nicht nur BewohnerInnen, sondern auch alle MitarbeiterInnen und Angehörige direkt oder indirekt betroffen.

Im Bundesland Steiermark ist das Corona-Virus bislang in 28 (von insgesamt 223) Pflegeheimen aufgetreten. Von den insgesamt bundeslandweit 147 Todesfällen im Zusammenhang mit Covid-19 stammen 82 Personen aus steirischen Pflegeeinrichtungen (Stand: 27.05.2020). Auch im südoststeirischen Pflegeheim Zerlach waren sowohl BewohnerInnen als auch Pflegende an Covid-19 erkrankt. In einem Interview mit Johann Platzer schildern Hausleiterin Brigitte Pichler und Pflegedienstleiterin Barbara Derler ihre beruflichen und persönlichen Erfahrungen während dieser Zeit.

Platzer: $\quad$ Frau Derler, Sie wurden vor einiger Zeit positiv auf das Corona-Virus getestet. Wie geht es Ibnen heute und wie ist es Ibnen während Ibrer Erkrankung ergangen?

Derler: $\quad$ Derzeit geht es mir gut, danke! Als ich vor einigen Wochen einen leichten Schnupfen bekam, dachte ich zuerst, dass meine Allergie wieder auftritt. Diese versuchte ich dann auch medikamentös zu behandeln. Plötzlich verlor ich dann aber auch meinen Geschmacks- und Geruchssinn. Das kam mir dann gleich verdächtig vor - und dieser Verdacht hat sich dann durch das Testergebnis bestätigt.

Platzer: $\quad$ Was waren Ibre größten Ängste und Sorgen, als Sie erfabren haben, dass auch Sie mit dem Corona-Virus infiziert sind? 
Derler: $\quad$ Meine erste Sorge galt dem Pflegeheim: Wer ist alles angesteckt? Wie gefährlich ist das Virus für die BewohnerInnen und KollegInnen? ... Dann hatte ich natürlich um meine Familie Angst: Habe ich sie schon infiziert? Wie wird das nun weitergehen? ... Und erst dann begann ich über mich selbst nachzudenken: Was passiert jetzt mit mir? Welche Symptome werden noch auftauchen? Ich hatte ja auch eine Nasennebenhöhlenentzündung und verspürte ständig Druck. Da kommen einem dann alle möglichen und unmöglichen Gedanken: Was ist, wenn sich die Verstopfung der Nasennebenhöhle löst und in Richtung Lunge wandert? ... Diese Zeit war alles andere als angenehm. Zum Glück konnte mich unsere Amtsärztin dann etwas beruhigen. Sie hat mir bei der Bewältigung der Situation sehr geholfen. Und die Symptome sind dann nach einigen Tagen auch wieder zurückgegangen.

Platzer: $\quad$ Sie standen dann ja 14 Tage unter Quarantäne. Wie hat Ihr Umfeld im Pflegeheim darauf reagiert?

Derler: $\quad$ Ich war ständig telefonisch oder durch elektronische Medien in Kontakt mit meinen KollegInnen. Diese haben mich immer mit aufmunternden Zusprüchen unterstützt. Allerdings hatten viele von ihnen auch selbst psychische Probleme, vor allem jene, die ebenfalls positiv auf Covid-19 getestet worden sind. Diesen KollegInnen versuchte ich dann von zu Hause aus per Telefon wieder Mut zuzusprechen. Es war und ist für alle KollegInnen eine herausfordernde und schwierige Zeit. Wir versuchen uns immer wieder gegenseitig Stütze und Halt zu geben.

Platzer: $\quad$ Nun aber der Reihe nach: Sie sind als Pflegedienstleiterin für 26 MitarbeiterInnen verantwortlich. Wann ist Ihnen eigentlich zum ersten Mal bewusst geworden, dass es aufgrund eines massiven Anstiegs von Covid-19-Erkrankungen zu einer Pandemie kommen könnte, und welche Gedanken gingen Ibnen damals durch den Kopf?

Derler: $\quad$ Es ist mir ziemlich schnell bewusst geworden, dass dieses Virus für unser Pflegeheim eine große Bedrohung werden könnte. Zunächst hat sich die mediale Berichterstattung ja vor allem um die Krankenhäuser und Arztpraxen und nicht um die Pflegeheime gedreht. Eine Arztpraxis kann geschlossen werden. Aber was ist mit einem Pflegeheim? Das kann 
man doch nicht so einfach von heute auf morgen zusperren! Ich kann ja nicht die Angehörigen anrufen und sagen: „Ihr müsst jetzt sofort eure Verwandten abholen!“ All das und noch vieles mehr ging mir damals durch den Kopf.

Platzer: $\quad$ Frau Pichler, Sie leiten seit etwa 14 Jahren das Pflegeheim Zerlach mit insgesamt 37 BewohnerInnen und über vierzig Mitarbeitenden. Was waren Ihre ersten Gedanken, als die WHO am 11. März 2020 die Covid-19-Krankheit zur Pandemie erklärt hat?

Pichler: Da waren wir schon mitten in den Überlegungen, was das für uns bedeuten wird und welche vorbeugenden Maßnahmen es für unser Haus zu treffen gilt, um Infektionen zu vermeiden. Es war für uns schon vor der WHO-Erklärung klar, dass das Virus uns ziemlich betreffen wird.

Platzer: $\quad$ Wann etwa war Ihnen das bereits bewusst?

Pichler: Die erste Dienstbesprechung dazu hat es schon Anfang März gegeben. Da hat es bereits erste Dienstanweisungen für die MitarbeiterInnen gegeben. Das Schwierige war, dass es keine validen Informationen und Pläne gab, wie wir im Haus damit umgehen sollen. Das war eine völlig neue Situation für alle Beteiligten.

Platzer: Welche Schutzmaßnahmen haben Sie zunächst für sich selbst und Ibre MitarbeiterInnen getroffen?

Pichler: Das Allererste war die Anweisung, die eigenen Kontakte zu kontrollieren und Abstand zu halten. Eine Mitarbeiterin war zum Beispiel zu dieser Zeit auf Urlaub in Tirol. Diese ist dann freiwillig 14 Tage zu Hause geblieben. Erst mit der Zeit erhielten wir dann erste Informationen sowie die Aufforderung, Schutzkleidung zu organisieren. Dann waren wir wochenlang damit beschäftigt, Desinfektionsmittel, Masken und Schutzkleidung zu organisieren. Das war aber äußerst schwierig, weil zu diesem Zeitpunkt kaum etwas verfügbar war. Auch unsere regelmäßigen Bestellungen wurden auf einmal nicht mehr geliefert.

Derler: $\quad$ Alle KollegInnen haben zunächst ihre notwendigsten persönlichen Sachen von zu Hause mit ins Pflegeheim genommen, denn wir mussten damit rechnen, dort für eine gewisse Zeit isoliert zu werden. Dann haben wir sofort im Eingangsbereich eine Art Barriere mit Desinfektionsmöglich- 
keiten aufgebaut, und alle BesucherInnen wurden von uns registriert. Und am 12. März wurde das Pflegeheim dann für Besuche geschlossen. Bei mir zu Hause musste ich erst einmal meine Familie von den zu setzenden hygienischen Schutzmaßnahmen überzeugen. Das war nicht einfach vor allem wenn es darum ging, die persönlichen Kontakte im Freundeskreis einzuschränken. Da kam es dann auch zu der einen oder anderen Meinungsverschiedenheit.

Platzer: Von wem sind denn die ersten Anweisungen gekommen?

Pichler: $\quad$ Also die ersten Informationen bekamen wir über die Medien. In weiterer Folge erfolgte dann ein Austausch mit anderen Pflegeheimen bzw. KollegInnen. Die ersten offiziellen Informationen sind dann erst um den 11. März vom Land Steiermark gekommen. Das war aber dann bereits die Aufforderung, die Pflegewohnhäuser quasi zu schließen und alle persönlichen Kontakte möglichst einzuschränken. Dann sind auch innerhalb der Caritas zweimal wöchentlich virtuelle Konferenzen eingerichtet worden, in denen sich die Haus- und PflegedienstleiterInnen austauschen konnten.

Platzer: Wie geht man in so einer Situation persönlich mit der Angst vor dem Virus um?

Pichler: Ich hatte nicht viel Zeit, mich zu ängstigen, da ich ständig mit Organisationsarbeiten und Sonstigem beschäftigt war. Ganz schwierig war es für mich auf jeden Fall, die Kontakte mit meiner Familie einzuschränken. Die Verhängung des allgemeinen Lockdowns war dann irgendwie sogar hilfreich. Zuvor konnte ich meinem Umfeld nämlich nur schwer erklären, warum ich ihnen gegenüber derartig auf Distanz gehe bzw. gehen muss.

Derler: $\quad$ Einmal besser, einmal schlechter. Am meisten hat mir in dieser Zeit, wie bereits erwähnt, der Austausch mit den eigenen KollegInnen geholfen; aber auch Kontakte mit KollegInnen aus anderen Pflegeheimen waren äußerst hilfreich. Ich habe dann auch gleich gemerkt, dass mir Gespräche mit Außenstehenden, d.h. mit Menschen, die keinen Einblick in den Alltag eines Pflegeheimes haben, wenig bringen, weil sich einfach niemand in meine Lage hineinversetzen konnte. 
Platzer: Wie kann man in Zeiten maximaler Unsicherheit Sicherheit geben? Und was heißt vor allem "in so einer Situation Vorbild sein"?

Pichler: Diese Frage habe ich mich auch oft gestellt. Ich habe einfach immer versucht, offen über die zu setzenden Maßnahmen zu sprechen. Darüber hinaus habe ich immer die MitarbeiterInnen nach ihrer Einschätzung und Meinung gefragt. Vorbild sein heißt für mich in solchen Situationen, mich mit der Lage ausführlich auseinanderzusetzen, transparent und offen über die Herausforderungen zu reden und mich möglichst nicht aus der Ruhe bringen zu lassen. Es hilft auch, wenn man sagt: „Ich bin mir auch nicht sicher, wie seht ihr das und was machen wir jetzt?" Das habe ich oft gesagt. Das heißt: Es kommt wahrscheinlich auf die Form des Umgangs mit Unsicherheiten an.

Derler: $\quad$ So banal es klingen mag, aber man muss in solchen Situationen einfach „funktionieren“ und mentale Stärke zeigen und persönliche Dinge müssen hintangestellt werden. Man braucht dafür nicht nur einen guten Background - also eine intakte Familie und Freunde -, sondern auch psychische Reserven.

Platzer: $\quad$ Woran orientierten Sie sich bei den Entscheidungen im Haus? Gab es so etwas wie Leitfäden oder Krisenhandbücher?

Pichler: Das erste Krisenhandbuch haben wir am 30. März bekommen, und zwar von der ARGE Tirol. Wir hatten das „Glück“ - zumindest aus unserer Perspektive -, dass es in anderen Pflegeheimen bereits früher als bei uns zu Erkrankungen gekommen war. In Tirol hatte man bereits sehr früh ein Krisenhandbuch für die Tiroler Pflegeheime erstellt, und das hatten sie dann österreichweit zur Verfügung gestellt. Dieses war auch das informativste und praxisrelevanteste. Der erste Leitfaden vom Land Steiermark ist dann am 17. April erschienen, und das Krisenhandbuch der Caritas überhaupt erst Ende April. Das war für uns alle viel zu spät, denn der 17. April war genau jener Tag, an dem wir erfahren haben, dass eine erste Bewohnerin von uns positiv auf Covid-19 getestet worden ist. Das heißt, das Virus muss da schon etwa 14 Tage vorher in unserem Haus gewesen sein. Die betroffene Bewohnerin ist nach Ostern ins Krankenhaus transferiert und zunächst negativ auf das Virus ge- 
testet worden. Weil sich ihre Symptome nicht gebessert haben, wurden noch weitere Tests durchgeführt, die dann positiv waren. Sie wurde dann vom Krankenhaus auf die Covid-Station verlegt, wo sie dann nach einigen Tagen verstorben ist - mit ziemlicher Sicherheit aufgrund des Corona-Virus.

Platzer: $\quad$ Sind dann alle MitarbeiterInnen und BewohnerInnen sofort getestet worden?

Pichler: Ja! Als klar war, dass die Bewohnerin an Covid-19 erkrankt war, hat sich sofort auch die Amtsärztin gemeldet, und dann sind alle getestet worden. Es ging dann auch sofort um die Kontaktpersonen-Verfolgung, also um die Frage, wer innerhalb eines bestimmten Referenz-Zeitraumes mit welchen Personen Kontakt hatte.

Platzer: Wie ist dann weiter vorgegangen worden?

Pichler: Dann kam es zu einer Klassifizierung der Personen, die mit der Erkrankten Kontakt hatten, in die Risikogruppen eins, zwei und drei. Alle, die zur Risikogruppe eins gehörten, mussten sofort nach Hause in Quarantäne gehen. Davon waren dann gleich zwei Pflegeteams betroffen. Wir arbeiteten damals nach einem Pandemie-Dienstplan, indem wir die Pflegepersonen in drei fixe Gruppen eingeteilt hatten, um Kontakte untereinander so gut es geht zu reduzieren bzw. zu vermeiden. Auch die Wohnbereiche wurden sofort getrennt. Das hat sich letztlich als sinnvoll erwiesen, da der Großteil der MitarbeiterInnen wieder nach einer bestimmten Zeit aus der Quarantäne zurückkehren konnte, da sie zum Glück nicht infiziert waren.

Platzer: Wie viele MitarbeiterInnen und BewohnerInnen wurden positiv auf das Corona-Virus getestet?

Pichler: In Summe wurden drei MitarbeiterInnen, inklusive der Pflegedienstleiterin, und zwei BewohnerInnen positiv getestet.

Platzer: Welche Maßnahmen wurden nach den ersten positiven Testergebnissen gesetzt?

Pichler: Als wir von einem weiteren positiven Testergebnis einer über 90-jährigen Bewohnerin in Kenntnis gesetzt worden sind, war diese noch bei uns im Haus. Sie befand sich damals in einem schlechten gesundheitlichen Zustand. In un- 
seren Augen war sie sterbend. Die Amtsärztin empfahl umgehend eine Verlegung auf die Covid-Station eines Krankenhauses, um das Ansteckungsrisiko zu verringern. Das war eine kritische Situation, weil wir uns alle fragten, ob für diese Bewohnerin eine Verlegung ins Krankenhaus überhaupt noch zumutbar sei. Nach mehreren Gesprächen kamen wir gemeinsam überein, dass die Bewohnerin die erste Nacht noch bei uns im Pflegeheim verbringen solle - natürliche unter besonderen Schutzmaßnahmen. Das Problem war hier die Risikoabwägung. Welcher Wert geht vor: die Gesundheit der restlichen MitbewohnerInnen oder die Situation der betroffenen Bewohnerin? Dieser konnte in dieser Situation einfach kein Transfer zugemutet werden, da sie einen solchen wahrscheinlich nicht überlebt hätte.

Platzer: Wie ging es dann weiter?

Pichler: Am nächsten Morgen ging es ihr dann deutlich besser. Sie hatte die erste Fieberkrise also gut überstanden. Sie war dann wieder ansprechbar und agiler. Dann versuchten wir wieder abzuwägen. Letztlich sind wir dann gemeinsam mit der Amtsärztin zur Entscheidung gekommen, dass sie aufgrund ihres verbesserten Allgemeinzustandes auf die CovidStation verlegt werden soll, obwohl ihr Hausarzt dagegen war. Im Nachhinein betrachtet war das von uns die richtige Entscheidung, weil die Patientin dann eine adäquate Behandlung bekommen hat und nach einiger Zeit wieder gesund zu uns zurückkehren konnte.

Platzer: Warum hat sich der Hausarzt gegen eine Verlegung der betroffenen Patientin auf die Spezialstation ausgesprochen?

Pichler: Der Hausarzt war deshalb dagegen, weil in diesem Fall ein älterer Mensch einem jüngeren das Bett wegnehmen würde und dieser dann womöglich an Covid-19 sterben müsste. Das war die Ansicht des Hausarztes - und diese Aussage hat uns alle sehr betroffen gemacht.

Derler: Ja! Ich kann mich noch ganz genau auf das Zitat dieses Hausarztes erinnern: „Diese Patientin ist über 90 Jahre alt. Wenn wir sie jetzt ins Krankenhaus bringen, dann nimmt sie ja nur einem 53-jährigen Patienten das Bett weg.“ Das ging dann schon in Richtung Triage, obwohl wir in der Steiermark ja nie einen Engpass an Spitalsbetten hatten. Das 
habe ich als äußerst negativ in Erinnerung. Wir haben, wie bereits erwähnt, die Verlegung ins Krankenhaus dann aber trotzdem veranlasst. Heute geht es dieser Patientin aufgrund der Spezialbehandlung im Krankenhaus wieder gut.

Platzer: $\quad$ Wie war im Allgemeinen die Zusammenarbeit mit den Hausärztinnen und Hausärzten?

Pichler: Zunächst muss erwähnt werden, dass wir mehrere Hausärztinnen und Hausärzte haben. Diese mussten sich - wie alle anderen auch - natürlich auch erst Informationen verschaffen und orientieren, wobei die Verunsicherung überall deutlich zu spüren war. Die Zusammenarbeit war also vorsichtig ausgedrückt - sehr unterschiedlich. Eine Hausärztin war sehr bemüht um uns und hat uns auch wohlwollend unterstützt. Von anderen Ärzten fühlten wir uns aber im Stich gelassen. Dabei möchte ich eines unbedingt erwähnen: Meines Erachtens sind viele BewohnerInnen einfach schlechter behandelt worden, weil die Hausärzte viel seltener ins Haus gekommen sind. Sie haben eine Zeit lang einfach keine Visiten mehr gemacht. Bei einigen PatientInnen wäre meines Erachtens eine Verlegung ins Krankenhaus zur Abklärung von Symptomen, die nichts mit Covid-19 zu tun hatten, unbedingt notwendig gewesen. Das ist aber ausgeblieben. Das hat ganz bestimmt für einige BewohnerInnen negative Konsequenzen gehabt. Mehr möchte ich dazu nicht sagen, sonst rege ich mich zu sehr auf. Diese Frage möchte ich jetzt an die Pflegedienstleiterin weitergeben, weil das ja eigentlich ihr Bereich ist.

Derler: $\quad$ Da kann ich mich leider nur anschließen. Ich habe die $\mathrm{Zu}$ sammenarbeit als sehr schwierig empfunden! Die Hausärzte haben auch immer wieder versucht, Testungen hinauszuschieben bzw. zu verhindern. Als wir einen sogenannten Verdachtsfall hatten und die betroffene Person in einem sehr schlechten Zustand war, hat sich ein Hausarzt geweigert, eine Testung zu veranlassen. Ich habe dann selbst die Amtsärztin verständigt und durch sie eine Testung in die Wege geleitet. Als dann in weiterer Folge das bereits erwähnte „Screening“, also eine Testung aller BewohnerInnen durchgeführt werden sollte, wollte dies wiederum ein anderer Hausarzt verhindern. Die Hausärzte hatten einfach eine große Unsicherheit und wollten immer nur zuwarten. $\mathrm{Zu}$ - 
nächst sind einige sogar ohne Schutzkleidung zur Visite gekommen, und wir mussten sie dann erst dementsprechend ausstatten. Mit der Zeit sind sie dann, wie bereits erwähnt, kaum mehr ins Haus gekommen.

Platzer: Wie konnten die BewohnerInnen dann medizinisch versorgt werden?

Derler: $\quad$ Vieles ist dann nur mehr telefonisch abgewickelt worden. Wir mussten auch Fotos von sichtbaren Symptomen machen und die Bilder dann zu den Ärzten schicken. Ich hatte einfach den Eindruck, dass sie große Angst hatten, auch selbst angesteckt zu werden und dann ihre Ordination schließen zu müssen. Ein Arzt war auch eine Zeit lang in Quarantäne, weil er mit einer positiv getesteten Person im Rahmen eines Einsatzes Kontakt hatte.

Platzer: Und wie hat die Zusammenarbeit mit der Sanitätsbehörde funktioniert?

Pichler: Die Kooperation mit der Sanitätsbehörde und der Bezirkshauptmannschaft hat bestens funktioniert. Da haben wir sehr viel fachliche Beratung hinsichtlich zu setzender Maßnahmen bekommen. Sie waren auch immer für uns erreichbar, sowohl in der Nacht als auch an den Wochenenden. Diese Unterstützung war hervorragend!

Derler: Das kann ich nur bestätigen. Sie haben uns auch immer wieder in unserem Handeln bestärkt und unterstützt. Wir hatten einfach das Gefühl, die Amtsärzte stehen immer hinter uns. Sie haben sich nicht nur für Testungen ausgesprochen, sondern auch dafür, dass die zuvor angesprochene Patientin ins Krankenhaus gekommen ist. Die AmtsärztInnen hatten für mich auch eine psychologische Funktion.

Platzer: Wie war die Kooperation mit den Krankenhäusern?

Pichler: Das war oft schwierig, weil diese zu dieser Zeit natürlich strenge Aufnahmebedingungen hatten. Da hatten wir z. B. schon vor der ersten positiv getesteten Patientin einen Bewohner mit Lungenentzündung. Diesen hätten wir gerne testen lassen, und wir wollten ihn auch ins Krankenhaus zur Untersuchung und Behandlung bringen. Das hat das Krankenhaus jedoch abgelehnt. 
Platzer: Wie wurde eigentlich offiziell nach außen kommuniziert? Hat es da irgendwelche Kommunikationspläne oder Leitfäden gegeben?

Pichler: Nein, da hat es überhaupt nichts gegeben! Als die Infektionen bei uns im Haus bekannt wurden, habe ich viel mit der Bezirkshauptmannschaft telefoniert. Ich wusste zunächst ja nicht, wie man mit so einer kritischen Situation umgehen soll. Wir haben uns dann mit unserer Ansprechpartnerin auf der Bezirkshauptmannschaft ein gemeinsames Wording überlegt und auf eine Informationsschiene geeinigt. Es ist von den Medien bei uns aber niemand aufgetaucht. Da hat es viele schlaflose Nächte gegeben - vor allem bei unserem Bürgermeister, vermute ich, der ja als Obmann dem Pflegeund Betreuungsverein vorsteht und im Krisenfall Rede und Antwort hätte stehen müssen.

Derler: $\quad$ Ich möchte hier ergänzen, dass uns auch der Bürgermeister immer wieder unterstützt hat und sich um uns gekümmert hat.

Platzer: Kommen wir nun zu den Bewohnerinnen und Bewohnern: Inwiefern hat sich deren Alltag während der Krise verändert und was waren dabei die größten Herausforderungen?

Derler: $\quad$ Am Anfang war es so, dass viele BewohnerInnen die veränderte Situation gar nicht mitbekommen haben - vor allem Menschen mit Demenz. Andere wiederum empfanden die Lage als derart tragisch, dass sie meinten, es sei „wie im Krieg“. Dann gab es aber auch welche, die alle Maßnahmen als „unsinnig“ bezeichnetet haben. Als wir dann unsere Schutzmasken getragen haben, fragten uns wiederum einige BewohnerInnen, warum wir uns verkleideten usw. Die wahren Herausforderungen sind aber erst mit der Zeit entstanden.

Platzer: Welche zum Beispiel?

Derler: Das betraf vor allem Menschen mit Demenz. Hier war zunächst das große Problem, dass diese uns nicht erkannt haben, da wir ja alle Schutzkleidung und Masken getragen haben. Aber sie haben zumindest nicht ängstlich reagiert. Nach einer gewissen Zeit haben die meisten aber begonnen, sich zurückzuziehen. Auch der Mangel an sozialen Kontakten mit ihren Angehörigen und jener an Bewegung hat sich 
bei vielen negativ ausgewirkt - sowohl körperlich als auch psychisch.

Platzer: $\quad$ Ist es dadurch auch zu vermehrten aggressiven Verhaltensformen gekommen?

Derler: $\quad$ Nein, eigentlich nicht. Einige BewohnerInnen haben mit Rückzug reagiert.

Platzer: Pflegerisches Handeln vollzieht sich „am Leib ebenso wie mit dem Leib". Wie kann man sich das durch den plötzlich geänderten Arbeitsalltag (Tragen von Schutzkleidung und Masken, Einschränkungen persönlicher Kontakte etc.) eigentlich vorstellen, vor allem wenn tagtäglich das Voneinander-Abstand-Halten gefordert wird?

Derler: $\quad$ Bei der Pflege gibt es kein Abstandhalten - Pflege kann nur mit Körperkontakt und „Zuwendung“ passieren! Die einzigen „Barrieren“ sind unsere Schutzmasken, Handschuhe und Einwegschürzen. Die Pflegeperson kann sich auch dadurch schützen, dass sie versucht, nicht angehustet oder angespuckt zu werden - was allerdings nicht immer möglich ist.

Platzer: Welche Angebote konnten den BewohnerInnen weiterhin gemacht werden? Auf was musste verzichtet werden?

Derler: Wir versuchten den Tagesablauf zunächst so gut es ging aufrechtzuerhalten. Allerdings mussten wir natürlich die Aktivierungen der BewohnerInnen sowie die Gruppen- und Unterhaltungsprogramme einschränken. Wir versuchten dann, den Schwerpunkt auf Einzelaktivierungen zu legen. Viele durften dann ja ihr Zimmer oder ihren Wohnbereich gar nicht mehr verlassen. Somit war das gemeinsame Essen im Speisesaal oder im Bereich des Wintergartens auch nicht mehr möglich. Der sonstige Tagesablauf, wie regelmäßige Pflege und Mahlzeiten, blieb natürlich gleich.

Platzer: War es zu dieser Zeit eigentlich möglich, dass BewohnerInnen, die an einer schweren Krankheit litten, von ibren Angehörigen besucht werden konnten?

Derler: Ja! Wir haben immer allen Angehörigen von BewohnerInnen, die palliativ betreut wurden oder sterbend waren, den Zutritt ins Pflegeheim ermöglicht - natürlich unter Beachtung der notwendigen Schutzmaßnahmen. 
Platzer: $\quad$ Hat es hinsichtlich dieser Besuchsmöglichkeiten bestimmte Empfehlungen oder Richtlinien gegeben?

Derler: $\quad$ Dafür hat es Richtlinien vom Bund, dem Hospizverein und dem Land Steiermark mit empfohlenen Maßnahmen gegeben; nach diesen sind wir vorgegangen. Ich habe die Aufrechterhaltung der Besuchsmöglichkeiten in diesem Kontext als sehr gut und sinnvoll erlebt. Von Angehörigen habe ich gehört, dass dies in Krankenhäusern nicht möglich war.

Platzer: $\quad$ Wie verlief in dieser Zeit eigentlich die Kommunikation mit den Angehörigen?

Pichler: Die Kommunikation mit den Angehörigen verlief primär über Telefon und E-Mail. Wir versuchten da immer umgehend transparent zu informieren. Als z. B. die erste Patientin positiv getestet wurde, haben wir alle Angehörigen sofort darüber informiert. Soweit es möglich und erlaubt war, haben wir zwischendurch auch ein Besucherfenster installiert.

Platzer: $\quad$ Und wie konnten die BewohnerInnen mit ibren Angehörigen Kontakt aufnehmen?

Derler: Die neuen technischen Kommunikationsmittel, wie zum Beispiel Videotelefonie, haben dabei eine ganz wichtige Rolle gespielt. Wir haben relativ schnell ein Tablet gekauft und so neue Kontaktmöglichkeiten geschaffen. Für Menschen mit Demenz waren Videotelefonate natürlich auch irritierend. Manche drehten sich weg, andere wiederum erschraken bzw. waren perplex, als sich ein vermeintliches „Foto“ einer Angehörigen plötzlich zu bewegen begann. Da gab es viele amüsante, aber auch rührende Szenen. Für viele war doch wieder das Telefon das geeignetere Kommunikationsmittel.

Platzer: Was waren für die Angehörigen die größten Herausforderungen?

Pichler: Die größte Herausforderung war natürlich der fehlende physische Kontakt mit den BewohnerInnen. Ganz besonders schlimm war es da für einige getrennte Ehepaare. Und manchen Angehörigen hat auch der plötzliche „Kontrollverlust" zu schaffen gemacht.

Platzer: Was meinen Sie damit? 
Pichler: $\quad$ Es gibt bzw. gab einfach immer ein paar Angehörige, die zuvor oft bei uns im Haus waren, um alles überwachen und kontrollieren zu können. Das war dann auf einmal nicht mehr möglich. Diese Angehörigen haben uns während dieser Zeit dann auch die größten Schwierigkeiten bereitet.

Derler: Wir haben aber auch viele nette und aufmunternde Briefe bekommen. Die meisten Angehörigen waren sehr verständnisvoll und kooperativ, aber es gab natürlich auch welche, die sich mit den aufgestellten Regeln und Maßnahmen schwertaten bzw. immer noch tun.

Platzer: $\quad$ Sind Sie da auch auf Unverständnis über die rigorosen Schutzmaßnahmen gestoßen?

Pichler: Ja! Es hat einige Angehörige gegeben, von denen wir immer wieder kritisiert worden sind. Diese haben es einfach nicht verstanden, warum diese Schutzmaßnahmen gesetzt werden mussten - und sie verstehen es bis heute nicht. Was hierbei von niemandem bedacht wird: Wenn ich mich infiziere, dann betrifft das ja nicht nur mich, sondern mein gesamtes privates und berufliches Umfeld, und zwar massiv. Dieses Bewusstsein sozialer Verantwortung ist bei vielen überhaupt nicht vorhanden. Der Großteil der Angehörigen hat die Maßnahmen aber verstanden und mitgetragen.

Platzer: $\quad$ Inwiefern haben sich dabei eigentlich die Beziehungen zwischen den BewohnerInnen, den Angehörigen und den Professionellen verändert?

Pichler: Am auffälligsten war zunächst, dass es plötzlich still im Haus geworden ist. Zuvor waren ja immer Angehörige und Besucher da, oder es hat irgendwelche Besprechungen gegeben. Das fiel auf einmal weg, und es herrschte plötzlich völlige Stille. Auch der Kontakt zwischen den BewohnerInnen musste massiv eingeschränkt werden. Auf einmal waren unsere MitarbeiterInnen, die noch dazu in ihren Schutzkleidungen wie „Aliens“ durch das Haus liefen, die einzigen Ansprechpersonen für unsere BewohnerInnen. Das war eine große Umstellung.

Derler: $\quad$ Ich finde, dass sämtliche Beziehungen viel enger geworden sind - vor allem jene mit den Angehörigen. Wir mussten ja ständig über die neuen Maßnahmen und Regelungen kommunizieren und informieren. Dadurch sind viel mehr Ge- 
spräche entstanden. Wir sind aber vor allem auch als Team viel mehr zusammengewachsen.

Platzer: Wie haben Sie dann die schrittweisen Lockerungen der Maßnahmen erlebt?

Pichler: Die Bewohner haben sich natürlich riesig darüber gefreut, dass sie endlich wieder in die Gemeinschaftsräume gehen und mit den BewohnerInnen aus den verschiedenen Wohnbereichen reden konnten. Die Lockerungen vollziehen sich aber äußerst langsam. Wir sind noch vorsichtig bei Veranstaltungen oder Gruppentreffen im Haus.

Platzer: $\quad$ Und wie sind die Lockerungsmaßnahmen bei den Angehörigen aufgenommen worden? Ist es dann eigentlich schnell zu einem Besucheransturm gekommen?

Derler: $\quad$ Nein. Das hat sich erst allmählich entwickelt. Viele Angehörige hatten zu Beginn noch Angst. Und wir merken nach wie vor, dass zwar die engeren Angehörigen öfters auf Besuch kommen, Nachbarn, Freunde oder die „weiteren Verwandten“ jedoch weit weniger oft. Diese Gruppe hält sich nach wie vor zurück.

Platzer: Gibt es schon erste Evaluierungen?

Pichler: Nein, das ist noch zu früh. Da fehlt uns jetzt einfach die Zeit dafür.

Platzer: $\quad$ Mit welchen wirtschaftlichen Auswirkungen rechnen Sie?

Pichler: Da haben wir dieses Jahr auf jeden Fall einen riesigen wirtschaftlichen Schaden. Wir mussten ja zusätzlich in die ganzen Schutzmaßnahmen investieren. Außerdem kamen und kommen immer noch Überstunden dazu und niemand konnte auf Urlaub gehen. Wie das alles zukünftig organisiert und finanziert werden soll - bzw. von wem -, das weiß ich zum heutigen Zeitpunkt alles noch nicht.

Platzer: Was werden Ihre nächsten Schritte sein?

Pichler: Wir brauchen demnächst eine intensive Aufarbeitung und Reflexion des Erlebten. Diese Wochen und Monate waren ja für alle eine traumatische Erfahrung - sowohl für die Mitarbeitenden als auch für die BewohnerInnen. Das ist die eine Ebene. Und auf einer zweiten Ebene müssen wir als Team und Organisation reflektieren: Was hat diese Krise mit uns gemacht? Welche Strukturen und Maßnahmen ha- 
ben wir als unterstützend und hilfreich erlebt und wo gibt es noch Entwicklungspotenzial? So etwas meistert man ja nur im Team. Um all das aufzuarbeiten, brauchen wir möglichst zeitnah eine professionelle Begleitung.

Platzer: $\quad$ Nun ein paar allgemeine Fragen: Bei den Diskussionen über Lockerungsmaßnahmen kommt auch immer wieder der Vorschlag, dass alte und schwache Menschen in der Isolation bleiben müssten, während der Rest der Gesellschaft wieder in den „normalen Alltag" zurückkehren soll. Wie beurteilen Sie eine solche „Strategie"?

Pichler: Das ist ein Ding der Unmöglichkeit. Das würden schon einmal die Angehörigen niemals akzeptieren. Eine längere Isolation ist niemals aufrechtzuerhalten. Das hätte ja massive Auswirkungen auf die Mitarbeitenden und die Angehörigen.

Derler: $\quad$ Am Anfang dachte ich mir: Diese Menschen müssen alle geschützt werden und sollen möglichst wenig mit anderen Personen in Kontakt kommen. Mittlerweile denke ich etwas anders darüber. Wenn ich mich nämlich in die Lage dieser Personen hineinversetze, dann würde ich auch nicht so lange in völliger Isolation leben wollen. Ich möchte auch nicht, wenn ich einmal alt bin, ständig bevormundet, sondern nach meiner Meinung gefragt werden. Das heißt, ich bin so einer Strategie gegenüber eher skeptisch eingestellt. Ältere Menschen verstehen es nicht, wenn sie mit fast niemandem in Kontakt treten dürfen - schon gar nicht, wenn es nur für sie und nicht für alle gelten soll.

Platzer: Diesbezüglich zeigt auch eine Reihe von Studien, dass sich soziale Isolation und Abschottung nicht nur negativ auf die psychische Gesundheit älterer Menschen auswirken, sondern auch mit einem deutlich höheren Sterblichkeitsrisiko einhergehen. Wie wirken sich Ihrer Erfahrung nach strenge Kontaktsperren auf die gesundheitliche Situation von hochbetagten und pflegebedürftigen Menschen aus?

Pichler: Man kann direkt zusehen, wie diese Menschen depressiv werden und „verfallen“. Unsere BewohnerInnen sind zwar - anders als in Privathaushalten - niemals ganz allein. Es ist bei uns ja immer jemand da. Aber wir können niemals die Beziehungen und die Menschen, die sie lieben, ersetzen. 
Das ist das große Problem. Dann kommt noch die ganze Langeweile dazu, die mindestens gleich schlimm wie eine Isolierung ist. Dieser massive „Abbau“ mancher BewohnerInnen war bereits nach drei Tagen deutlich wahrnehmbar. Viele haben auch gesagt: „Ich will lieber sterben, als dass ich meine Angehörigen nicht mehr sehe."

Derler: $\quad$ Dem kann ich nur zustimmen. Nach einiger Zeit habe ich zu Brigitte gesagt: „Wenn wir diese Isolations-Maßnahmen bei unseren BewohnerInnen noch länger durchziehen müssen, dann sterben sie entweder am Corona-Virus oder an Einsamkeit.“

Platzer: $\quad$ Während der "Corona-Krise" werden ältere Menschen vor allem als "Risikogruppe" und als „zu Beschützende und Schwache" wahrgenommen. Was löst dies Ihres Erachtens bei älteren Menschen aus, wenn sie ständig damit konfrontiert werden?

Derler: Das löst bei diesen Menschen meines Erachtens ein ganz starkes Gefühl der Bevormundung aus. Das ist so, als wenn ich einem Kind verbieten würde, auf einen Baum zu klettern, da es ja herunterfallen könnte. Das kann man mit erwachsenen Menschen im Grunde genommen nicht machen. Alter darf kein Stigma sein, sondern muss Selbstbestimmung zulassen und wir - die Gesellschaft - müssen diese Selbstbestimmung unterstützen und fördern.

Platzer: $\quad$ Einige Gesundheitsorganisationen sehen in diesem Kontext die große Gefahr eines Rückschritts. Dieser könnte darin bestehen, dass sich negative Einstellungen gegenüber älteren Menschen verstärken und sich diskriminierende Praktiken einstellen könnten. Sehen Sie diese Gefahr auch?

Derler: Altersdiskriminierung gibt es schon jetzt in vielen Bereichen, und ich sehe hier tatsächlich eine Gefahr, dass diese zunehmen könnte.

Platzer: Wie könnte man dem entgegenwirken?

Derler: $\quad$ Indem man in der Gesellschaft viel mehr darauf hinweist und Altersdiskriminierung zum Thema macht. Nur untereinander darüber zu reden, das wird zu wenig sein. Dieses Thema muss viel mehr medienwirksam in der Öffentlichkeit thematisiert werden. Das gilt natürlich auch für alle anderen diskriminierten Gruppen. 
Platzer: Angenommen, es kommt zu einer zweiten sogenannten Welle oder zu einer neuen Pandemie: Welche Wünsche und Verbesserungsvorschläge würden Sie aufgrund der bisherigen Erfahrungen an das Gesundheitssystem herantragen?

Pichler: Wir wissen jetzt einfach alle zusammen viel mehr - auch sämtliche Behörden. Auf so eine Situation war ja niemand vorbereitet. Wir können aufgrund dieser Erfahrungen zumindest für unser Haus Rückschlüsse für die Zukunft ziehen. Jetzt gilt es zu analysieren, was gut funktioniert hat, was nicht funktioniert hat und wo unsere Grenzen sind. Der Inhalt vieler aktueller Krisenhandbücher mag zwar theoretisch richtig sein, aber für deren Umsetzung braucht man auch dementsprechende Strukturen und vor allem Personal. Wir brauchen mehr Personal! Es wäre zukünftig auch sinnvoll, dass ein bestimmtes Mindestausmaß von Schutzmaterial auf Lager gelegt wird. Das war ja aufgrund des weltweiten Bedarfs zunächst ganz schwer aufzutreiben.

Platzer: Wie lange hat es eigentlich gedauert, bis für alle MitarbeiterInnen einigermaßen ausreichend Schutzmaterial und Schutzkleidung organisiert werden konnte?

Pichler: Das hat gute vier bis fünf Wochen gedauert.

Platzer: Die jetzige Krisensituation bringt auch allgemeine Versäumnisse im Gesundheitswesen - vor allem im Kontext der Pflege - zum Vorschein. Glauben Sie, die jetzige Situation eröffnet auch eine Chance, die Herausforderungen der Pflege konstruktiv aufzugreifen und notwendige Veränderungen endlich anzugehen?

Pichler: Da gibt es viele Lippenbekenntnisse. Ich persönlich glaube erst dann daran, wenn diese Versprechungen tatsächlich umgesetzt werden. Die Frage dabei ist immer, wer politisch dafür verantwortlich ist und welche Prioritäten dabei gesetzt werden.

Derler: $\quad$ Sicherlich sehe ich es auch als Chance, aber ob diese genützt wird - das weiß ich nicht. 
Platzer: Welche strukturellen und finanziellen Verbesserungen sollten angesichts der bereits bestehenden kritischen Situation in der Langzeitpflege schon jetzt anvisiert werden, damit man nicht nur aus der Corona-Krise herauskommt, sondern damit es auch zu nachhaltigen Verbesserungen im Pflegebereich kommt?

Pichler: Nochmals: Wir brauchen mehr Personal! Das wird aber schon lange diskutiert, und es ist auch geplant, dass hier aufgestockt wird. Der Wille allein reicht aber nicht aus, denn es gibt nach wie vor einen Mangel an qualifizierten Fachkräften. Es braucht hier vor allem einen Ausbildungsschwerpunkt im Bereich Geriatrie und Altenpflege. Das hat man auch bei der Novellierung des Gesundheits- und Krankenpflegegesetzes wieder vernachlässigt. Da muss sich in den nächsten Jahren tatsächlich einiges tun, denn sonst kann zukünftig eine qualitative Pflege tatsächlich nicht mehr gewährleistet werden.

Derler: Darüber hinaus braucht es auch eine zentrale bundesweite Organisation und Finanzierung der Pflegevorsorge. In Österreich gibt es bekannterweise neun Bundesländer und jedes kocht sein eigenes „Süppchen“. So kann das nicht gehen! Dann muss die Situation der Beschäftigten verbessert werden: mehr Personal in den Gesundheitsbereichen, eine gerechte und angepasste Entlohnung für die Arbeit und Maßnahmen, die den Pflegeberuf attraktiver machen. Ich finde es z.B. ganz schlimm, dass nun ausländische Pflegekräfte in Zügen „verfrachtet“ werden und auf diesem Wege nach Österreich kommen, wo sie dann zunächst in Quarantäne gehen müssen und quasi eingesperrt werden. Das ist menschenunwürdig und erinnert mich an Kriegszustände. Diese Situation finde ich wirklich total schlimm. Diese Problematik sollte im Fokus stehen und da müssen Lösungen gefunden werden.

Platzer: Nehmen Sie auch etwas Positives aus dieser Zeit mit?

Derler: Ja, vor allem die Erkenntnis, dass es trotz extremer Herausforderungen weitergeht, und die Erfahrung, dass Krisen auch bewältigbar sind. Im Endeffekt geht man gestärkt aus der Krise hervor und ist für die Zukunft vielleicht schon besser gerüstet. 
Pichler: Natürlich! Für mich persönlich war es einmal der reinste „Selbsterfahrungstrip“. So eine Situation musste ich ja noch nie zuvor managen. Da lernt man einiges über sich selbst. Als extrem positiv habe ich auch den unglaublichen Zusammenhalt zwischen den MitarbeiterInnen in unserem Haus erlebt - und auch, wie diese mit der Krisen-Situation umgegangen sind. Das wurde von uns allen gemeinsam durchgetragen. Das war für mich äußerst beeindruckend, und das wird sich auch nachhaltig positiv auf uns alle auswirken.

Platzer: Vielen Dank für das Gespräch und weiterhin alles Gute! 
